\title{
Bogan bias: Addressing class-based prejudice in physician-patient interactions
}

\section{Mark Kennedy}

University of Melbourne

\section{Maeve Kennedy}

Northern Bay Health

\begin{abstract}
There is a widespread belief in the 'classlessness' of Australian society, despite strong evidence demonstrating the impacts of socioeconomic status (SES) on individuals' educational attainment, health, and mortality. Disparities in health care are also prevalent. The quality of communication between physicians and patients is associated with health outcomes and patient satisfaction, and we argue that this communication can be influenced by socioeconomic bias and prejudice. The majority of medical students in Australia are from backgrounds of high SES, and this is likely to influence their communication as physicians with patients from lower SES communities. In particular, mediatised representations of the Australian workingclass as 'the bogan,' and the acceptability of derogatory humour towards those perceived to be 'bogans' - in the absence of lived experience and understanding of lower SES life - can influence the attitudes, expectations, and behaviour of physicians working in low SES communities. To begin to address these biases, we recommend expanding cultural competence training to reflect a multidimensional understanding of culture that includes SES, and going beyond cultural competence to promote self-reflexivity and critical awareness of personal socio-cultural backgrounds, assumptions, and biases, in staff induction programs.
\end{abstract}


Keywords: General practice, physician-patient interaction, implicit bias, prejudice, socioeconomic status, working-class

\section{Introduction}

A widespread belief in the egalitarian, 'classless' nature of our society is held across Australia. In his budget reply speech as Leader of the Opposition in 2012, Tony Abbott declared he was "grateful that our country has normally been free from the class struggle that's raged elsewhere to other countries' terrible cost." However, despite the invisibility of class distinctions in mainstream public discourse and the political inconvenience and personal discomfort of deeper class analysis, social class - whether defined by income, education level, or occupation - has an ongoing impact on Australians' opportunities, education, health, and mortality. In Australia and across the world, health follows a social gradient, with the prevalence of disease and many health conditions decreasing incrementally as socio-economic status (SES) increases (Adler \& Ostrove, 1999; Marmot, Allen, Bell, \& Goldblatt, 2012; Marmot et al., 1991).

In addition, the quality of health care provision influences disparities in health outcomes, and this is also associated with SES (Raingruber, 2014). One of the primary ways in which health care provision is affected by SES is physician-patient interaction. Doctors, even those with egalitarian beliefs, communicate differently with patients from different social groups, including SES, racial/ethnic and stigmatised groups (Burgess, Fu, \& van Ryn, 2004; Verlinde, De Laender, De Maesschalck, Deveugele, \& Willems, 2012). Effective communication and satisfactory interactions between physicians and patients are a key part of ensuring high-quality health care, positive health outcomes, and full social inclusion (Aronson, Burgess, Phelan, \& Juarez, 2013; Kaplan, Greenfield, \& Ware, 1989). Societal discourses of class-based disparagement and derogatory humour, and a growing literature on bias and prejudice highlight some of the challenges physicians may face when working in lowSES communities. In this article, we examine key representations of the workingclass in contemporary Australia and explore how these may influence the biases, communication, and behaviour of medical professionals working in low-SES areas. 
We also offer some suggestions for how to reduce these biases and create more inclusive and effective physician-patient interactions.

For the purposes of this article, we define class and SES as "an individual's socioeconomic ranking in society" (Ridgeway \& Fisk, 2012, p. 132), and use the terms interchangeably. The scope of this piece necessitates certain limitations, and means we address disadvantage and social exclusion from a purely class perspective, ignoring the multiple and intersecting dimensions of status and disadvantage. We recognise the 'ethnicity blindness' in our article and that the implicit assumption that the majority of physicians and patients are White, ignores the diversity of Australian communities (including the proportion of overseas-trained doctors in our medical system, circumventing our discussion of Australian medical students). However, we feel that such a limitation is required to enable an appropriate examination of the influence of mediatised representations of the Australian working-class on physicians - as Campbell (2004) notes, "the figure of the bogan is conspicuous for its whiteness" (p. 9) - and for clarity of discussion. We acknowledge the potential dangers of this siloed thinking and are also conscious of the danger of perpetuating essentialist class-based thought through our discussion.

\section{Contemporary attitudes to the Australian working-class}

The strong belief in a 'classless' Australia continues despite growing inequality (Leigh, 2013; Nichols, 2011), with recent data estimating that 14.4 percent of Australians live in relative poverty compared with an OECD average of 11.3 percent (OECD, 2014). However, the invisibility of class is not a uniquely Australian phenomenon. Much literature highlights an enduring perception of egalitarianism 'we are all middle-class' - in the United States despite growing inequality (Fiske, 2011). Lawler (2005) argues that the limited acknowledgement of class distinctions and the "perceived decline in the significance of class itself" in the United Kingdom further legitimises the normalisation of 'the middle class' in social norms (p. 429).

The institutionalisation of middle-class attitudes and norms into broader social norms and practices (DiMaggio, 2012; Ridgeway \& Fisk, 2012; Stephens, Fryberg, \& Markus, 2012) and the continuing acceptance of neoliberal constructions of individual responsibility further add to the 'pathologisation' of the working-class (Lawler, 2005). It logically follows that common middle-class representations of the working-class are likely to inform or influence the attitudes of medical professionals 
working in low-SES communities. The primary contemporary representation of the Australian working-class is 'the bogan,' a group commonly perceived to be parallel to the American 'white trash' and the British 'chav' (Gibson, 2013; Loughnan, Haslam, Sutton, \& Spencer, 2013; Nichols, 2011). Bogan discourse reinforces class distinctions as cultural differences, without needing to use traditional class labels, and essentially functions as "denigration of the undereducated or sociallydisadvantaged" (Gibson, 2013, p. 63).

'The bogan' is a popular, often comedic, figure in pop-culture. It features in many Australian films and television programs, including The Castle and Kath \& Kim, and is often also employed in current affairs and news stories (Campbell, 2004; Gibson, 2013; Nichols, 2011). Bogan discourse is further normalised when it is used by public figures, such as Melbourne Lord Mayor Robert Doyle advising 'bogans' to "bogan it up at home" rather than in the city on New Year's Eve (Nichols, 2011, p. 71). Much discussion, particularly on the internet, refers to 'bogans' in the dehumanising 'it' form through an anthropological, David Attenborough-style narrative (Nichols, 2011; Pini \& Previte, 2013). A recent example of this narrative is the free-to-air television program Bogan Hunters, an "Australia wide hunt for our greatest bogans in their natural habitat," which aired as we were writing this article. Images of "bogans in their natural habitat" posted on the Bogan Hunters Facebook page include a photograph depicting two children within a binocular-style frame, with the caption "In this herd spotted in the urban wild of Australia, there can be seen examples of younglings of the bogan species with traditional hair." Bogan Hunters seems to have a considerable degree of popularity (evidenced by more than 133,600 'Likes' and significant engagement in the comments section of the Facebook page), however, there has also been media critique of the show and its classist narrative (Enker, 2014).

One of the consequences of these media narratives is that their representation of the working-class, similar to Spencer and Castano's (2007) analysis of pop-cultural depictions of the American poor as unintelligent, "dirty, violent, inbred, lazy, unkempt, carefree hillbillies" (p. 419), informs middle-class attitudes and beliefs. The bogan narratives help to construct a "common understanding" of the working-class, producing a "set of shared meaning about what working-class people are (and hence, what the middle classes are not, and could not be)" (Lawler, 2005, p. 431). This reproduces an understanding of working-class 
people as 'the Other' (Gibson, 2013; Nichols, 2011), further normalising the middleclass experience (Lawler, 2005).

The ease with which the "offhand" label of 'bogan' can be applied to people, activities and places, without acknowledgement or recognition of its inherent "class hatred" (Nichols, 2011, p. 72; 215) strengthens implicit perceptions of fundamental class distinctions. Instead of recognising class as a product of social and economic history and context, the term perpetuates the 'pathologisation' of the working-class through a language of culture, depersonalisation, and choice (Lawler, 2005). The popular media use of the 'bogan' figure and language indicates its social acceptability, and it is normalised in "casual conversations," where "[e]ven 'sighting' bogans on the street is a mediatised deployment of the bogan discourse" (Campbell, 2004 , p. 16). Social and faculty acceptability of stigmatisation or negative attitudes towards individuals on the basis of their demographic characteristics, such as weight and poverty level, is thought to influence medical students' attitudes and levels of implicit and explicit bias (Beagan, 2005; Phelan et al., 2013), and the 'bogan' discourse is likely to operate in a similar way.

However, there are alternative narratives of class-based difference in Australia, and we acknowledge the way in which our discussion in this article assumes a unidirectional perspective through focusing on attitudes of the middleclass towards the working-class and the way this reproduces exclusive modes of social thought. We note the two-way nature of class relationships in reality, for example, working-class attitudes towards the 'sin' of putting on airs (Skeggs, 2011), and the pride with which ownership of the 'bogan' label has recently been claimed and self-identified (Gibson, 2013; Rossiter, 2013). This pride and self-identification is highlighted by Paul Fenech, the creator and director of Bogan Hunters, who argues, "There are people who do classify themselves as bogans so I thought it would be a laugh because a lot of them are great characters. There's an optimism and an honesty and there's a real bogan culture and they seem cool with it" (McMahon, 2014).

We support David Nichols' argument of 'the bogan' as mythology rather than an active Australian identity. However, the physical existence or absence of 'the bogan' does not have an effect on our core argument: that mediatised constructions and representations of the working-class as 'the bogan' are likely to have an impact 
on the attitudes, biases, and expectations of those from other class backgrounds beginning work in a low-SES area.

\section{The class background of medical students}

Understanding the demographic trends in medical students' backgrounds can help to draw out potential attitudes and biases; higher education level tends to be associated with "more negative attitudes toward low-SES individuals" (Spencer \& Castano, 2007, p. 421). There is limited current published data examining the backgrounds of medical students in Australia; however, consistent evidence from the past twenty years shows that the majority of medical students tend to be from advantaged backgrounds, and this is proportionally higher than other university courses; similar trends are found in other Western nations (Dhalla et al., 2002; Fitzjohn, Wilkinson, Gill, \& Mulder, 2003; Wear \& Kuczewski, 2008).

In Australia in 1996, 57 percent of medical students were from high-SES postcodes and 10 percent were from low-SES postcodes, compared with 34 percent and 14 percent respectively of all tertiary enrolments; for comparison, in 1996, 25 percent of the Australian population lived in high-SES areas and 25 percent lived in low-SES areas (AMWAC, 1997, p. 29). There continue to be significant barriers to higher education for students from low-SES communities in Australia, for medical and other tertiary courses. A study commissioned by Universities Australia found that in 2008, "high SES students continue to be three times more likely to go to university as those from low SES backgrounds," a pattern that is largely unchanged since 2001 (UA, 2008).

The under-representation of medical students from low-SES communities and the over-representation of medical students from high-SES backgrounds have implications for these students' attitudes towards and communication with individuals from low-SES groups, as well as the depth of their understanding of the social determinants of health (Beagan, 2003, 2005). Growing up in a high-SES environment can result in a form of 'solipsism' fundamentally affecting the way in which one views and interacts with other individuals and groups (Geiger \& Jordan, 2013). Wear and Kuczewski (2008) suggest that the social contexts of most medical students, "most often the in-group — white, economically privileged, and soon to-bedoctors," mean that they are "without the natural feeling of "oneness" with the circumstances of many of their patients' lives" (p. 642). One could assume that in the 
absence of lived experience and exposure to the low-SES context, mediatised representations of 'the bogan' would significantly inform many medical students' views and attitudes towards low-SES groups.

\section{Class bias and prejudice}

Group categorisation is considered fundamental to human interaction, acting as a filter for information overload by allowing individuals to make rapid decisions about groups of people based on stereotypes (Balsa \& McGuire, 2003; Major, Mendes, \& Dovidio, 2013; Phillips, 2010). This cognitive process is valuable for medical practitioners, who are expected to make rapid and complex decisions in their interactions with patients, however, it can also lead to over-generalisations, stereotype-consistent biases, and discrimination (Major et al., 2013, p. 515; Phillips, 2010; van Ryn \& Burke, 2000).

In general, societal out-groups, including 'the poor,' are considered to be more hostile, less intelligent, less trustworthy, and less motivated than in-groups, and they elicit more feelings of disgust and contempt (Fiske, Cuddy, \& Glick, 2007). Recent research indicates that out-groups may also be viewed as less biologically evolved than in-groups: a study of Australian undergraduates found that "people think that the attributes that distinguished low from higher-SES people are the same as those that distinguish apes from humans" (Loughnan et al., 2013, p. 5).

Research has also found that physicians often hold biases toward those in low-status social groups, and rate low-SES patients as having lower self-control, rationality, and intelligence than middle- and upper-SES patients (Major et al., 2013; van Ryn \& Burke, 2000). Lower-SES patients are considered to be "less likely to be compliant with cardiac rehabilitation, less likely to desire a physically active lifestyle, less likely to have significant career demands, less likely to have responsibility for care of a family member and more likely to be ... at risk for inadequate social support" (van Ryn \& Burke, 2000, p. 821). Similar biases and negative affective responses have been found for medical students. Second-year medical students watching a video of an actor playing a low-SES patient were "less inclined to want that patient in their practice" than those watching a video of the same actor playing a high-SES patient (Woo, Ghorayeb, Lee, Sangha, \& Richter, 2004, p. 1915).

Implicit bias, bias that operates unconsciously and unintentionally influences behaviour, is thought to be particularly influenced by cultural norms and media 
coverage (Major et al., 2013; Rudman, 2004). It would seem logical to expect that the derogatory media representation of the Australian working-class might significantly influence the attitudes and behaviour of individuals from higher-SES backgrounds towards work with low-SES groups. Additionally, the social acceptability of class bias compared with other forms of social prejudice, supports the idea that there is low external pressure to address this bias and may influence medical students' beliefs about the acceptability of this bias and subsequent discriminatory or exclusive behaviour (Phelan et al., 2013).

System justification theory posits that people endorse stereotypes that support the status quo of the social hierarchy, and people tend to assume that social status reflects merit and competence (Fiske et al., 2007). Approximately 50 percent of respondents in a recent American study believed that strong or weak personal effort dictated whether people are wealthy or poor (Spencer \& Castano, 2007), and perceived causes of poverty are likely to be associated with attitudes towards those living in poverty (Wear \& Kuczewski, 2008). System justification thinking can legitimise and encourage differential treatment and perpetuate stereotypes that justify this treatment, such as patients' noncompliance behaviour (Major et al., 2013). In the stereotype content model of cognition, individuals make judgements of social groups based on perceived status and competence, treating these dimensions as linked, although status is based on demographic factors and competence is based on personal traits (Fiske et al., 2007; Harris \& Fiske, 2006). These theories help to explain the varying perceptions of low-SES patients as inherently less competent and less intelligent than higher-SES patients, irrespective of structural barriers to health behaviour.

\section{The impact of class bias on health}

These stereotypes and biases can have significant consequences for inter-class and physician-patient interactions, which in turn can affect health care and health outcomes. Bias and/or prejudice held by physicians is one of the most common interpretations of disparities in medical encounters (Balsa \& McGuire, 2003), and as Verlinde et al. (2012) note, a "prerequisite for equity in health is equity in health care" (p. 1). The quality of communication between physician and patient is consistently associated with patient satisfaction and compliance, which affects overall wellbeing and health outcomes (Bensing, 1991; Verlinde et al., 2012; Willems, De 
Maesschalck, Deveugele, Derese, \& De Maeseneer, 2005). Data from the United States reveals that 30 percent of individuals from the lowest income group feel 'disenfranchised' in their health care experiences, compared with 20 percent from the highest income group; and that a high proportion of patients (55 percent of Chinese individuals and 40 percent of White individuals) feel that their doctors do not "understand their background and values" (Raingruber, 2014, p. 142). In another study examining the connection between SES and health literacy, patients who were considered to be 'poor' or 'near-poor' were less likely to report that physicians always explained things so that they understood, compared with patients from higher income brackets (DeVoe, Wallace, \& Fryer, 2009).

The predominantly higher-SES background of physicians can influence their communication style and their ability to effectively interact with patients from lowerSES backgrounds; this includes physicians' awareness of the effectiveness of their interaction (Epstein, Taylor, \& Seage, 1985). Physicians' interactions with lower class patients tend to be characterised by "less positive socio-emotional utterances and a more directive and less participatory consulting style ... [including] significantly less information giving [and] less directions" (Willems et al., 2005, p. 139), and they tend to adopt "less patient-centred communication with patients they believe will not comply with recommendations" (Phelan et al., 2013, p. 1). Physicians with higher levels of implicit bias towards a certain (racial) group have been perceived as "less warm and friendly" by members of that group, without recognising differential behaviour (Major et al., 2013, p. 517). People tend to be more able to read affective signals appropriately from individuals within their in-group, and less familiar affective responses can be misinterpreted as disinterest or disengagement (Major et al., 2013; Willems et al., 2005). Different cultural backgrounds and norms also inform behaviour and "ideas and practices about how to be a culturally appropriate person" (Stephens et al., 2012, p. 89).

The social class hierarchy can effectively institutionalise rank-based interactions, with interpersonal communication following a pattern of deference and assertion (Goodman, 2011; Ridgeway \& Fisk, 2012). The social contexts of the participants, and the status and power differentials at play, thus have an enormous capacity to influence communication in a consulting room, and it is important to remember the two-way nature of this interaction. As well as physicians' biases and behaviour, patients' active participation in the consultation and their "expectations 
and apprehensions" - as well as the interplay between these factors - also influences the quality of communication (Balsa \& McGuire, 2003; Jensen, King, Guntzviller, \& Davis, 2010; Major et al., 2013, p. 519). The levels of trust, participation and satisfaction held by patients affect compliance and commitment to treatment (Major et al., 2013), and it is thus important for health care providers to be aware of the two-way communication and to work towards building a relationship based on mutual trust and respect.

The absence of this nuanced understanding of cross-status communication, and the presence of class-based biases, can lead to differential quality of care and disparities in health outcomes (Phelan et al., 2013). In turn, discrimination has a negative impact on an individual's health, wellbeing, and feelings of self-esteem and social inclusion (Major et al., 2013; VEOHRC, 2014, p. 5). Negative stereotypes, for example, the belief that patients from low-SES groups are less likely to comply with prescribed treatment, can become self-fulfilling and perpetuate treatment disparities (Balsa \& McGuire, 2003). Stereotypes of incompetence and lack of motivation reinforce incorrect assumptions around patient's reasons for non-compliance, including transport disadvantage and physical safety within the community, their understanding of healthy behaviours and appropriate preventive care, and their literacy and numeracy levels.

Biases and assumptions, whether they are intentional or unconscious, have a significant impact on the quality of interpersonal communication, which, in the context of health care provision, can have significant and real consequences on health behaviours and subjective and objective health outcomes. Understanding the ways that these cognitive processes are influenced by media and pop-cultural representations of various SES groups is also critical, and in this article we have tried to outline various pathways through which this occurs in contemporary Australia. As noted earlier, it is also important to recognise the bidirectional nature of social biases and assumptions, and to understand the effect that patients' biases and assumptions may have on interpersonal relationships in a health care setting. A self-reflexive understanding of the class and cultural contexts of each party in the consultation room is crucial for building a more effective, more inclusive, and more equitable health system. 


\section{Overcoming prejudice and promoting inclusion in physician-patient interactions}

Cultural competence, being "aware of cultural differences and able to actively solicit and respond to differences" (Voss-DeMeester, McCullough, Cook, El-Shamaa, \& Chin, 2014, p. 3), encompasses SES and other demographic markers in much literature (Kelly, 2012a, 2012b; Voss-DeMeester et al., 2014). Unfortunately, Australian literature overwhelmingly considers culture to be predominantly race- or ethnicity-based, with the 85-page 'Cultural Competency in Health' guide from the National Health and Medical Research Council containing only two references to socioeconomic factors (NHMRC, 2006). Multidimensional cultural competency is essential for health care providers working in low-SES communities, and can help to mitigate the unconscious attitudes and biases discussed above. Inadequate cultural competency training and the impact of (unconscious) bias while students are at medical school or come into contact with people from lower-SES groups - including "informal influences" in medical schools, such as peer-to-peer interaction and "derogatory humor" - can lead to the development of misconceptions and reinforcement of negative stereotypes (Kelly, 2012a; Phelan et al., 2013, p. 2; Rudman, 2004; Wear \& Kuczewski, 2008). This may result in lower quality, unsatisfactory physician-patient communication and inadequate attention to the patient's psycho-social wellbeing. Constructive cultural competency training should develop physicians' self-awareness (Goodman, 2013), and encourage them to recognise their personal cultural upbringings and norms and develop a pluralistic understanding of culture and discrimination and their effects (Kelly, 2012b; Raingruber, 2014).

However, to properly address health disparities and intersecting dimensions of social inclusion it is necessary to go beyond cultural competency training (VossDeMeester et al., 2014). Successful cross-class interactions include: an individual's positive feelings towards the other party and themselves, the individuation of the other (seeing them as more than the sum of stereotypes and representations of their social groups), cooperation, respectful behaviour throughout the interaction, and reflections after the interaction (DiMaggio, 2012). Although medical students in Australia today receive extensive training in effective communication, an awareness of some of the unique issues that arise in cross-class interactions remains important, and this could be included in their training and workplace induction programs. As an 
example, the staff induction program at Northern Bay Health, a privately owned network of general practices operating in low SES communities in a regional Australian city, includes a comprehensive induction manual that provides an introduction to the community, the key health challenges and barriers to health, and the current community development initiatives, highlighting examples of positive community action and resilience (Kennedy, 2013). It discusses the expanded role of physicians in low SES communities, outlining common assumptions and misconceptions, as well as the components of positive and effective physicianpatient communication in such communities.

There is a wealth of research in the fields of medicine, social psychology, and education, discussing methods to reduce bias and increase social justice. An understanding of the issues is the first step to addressing bias, and as such, the provision of information to medical students and health professionals is fundamental. The provision of information about structural and environmental causes of ill health can reduce implicit and explicit bias, and information challenging the social acceptability of negative attitudes towards certain social groups is recommended (Phelan et al., 2013). An awareness of explicit and implicit bias and the development of evidence-based guidelines for consultations in lower SES settings may reduce the scope for biases to interfere with treatment (Major et al., 2013).

Health professionals' ongoing critical self-awareness is crucial to effective and inclusive cross-class interaction. Although the Equal Opportunity Act doesn't officially refer to SES as a dimension of discrimination, the Victorian Equal Opportunity and Human Rights Commission notes the importance of self-reflection and selfawareness as a 'positive duty' of health professionals to ensure non-discriminatory and equitable medical practice (VEOHRC, 2014). Reductions in explicit bias can occur through education and increased self-awareness, and implicit orientations can be reduced by personal contact with individuals from the group for which the bias holds, if this contact is undertaken alongside adequate education and preparatory work (Rudman, 2004).

\section{Conclusion}

While we acknowledge that localised information-based policies and individual-level reflective practices in isolation are unlikely to challenge broader social norms and 
address systemic bias and discrimination (Balsa \& McGuire, 2003), it is a step towards increasing inclusion in our society and creating a more accepting and respectful national discourse, one that encompasses social class realities as another dimension of contemporary life but does not accept them as a basis for prejudice, derogation, and inequality. As Rudman (2004) notes, "[t]o the extent that societal evaluations color implicit biases, a more inclusive society should reduce them" ( $p$. 138), and we must all be conscious of the effect of negative attitudes towards others on the basis of their belonging to a particular social group, even those expressed in casual conversations, in perpetuating exclusive and offensive social norms.

\section{References}

Abbott, T. (2012). Leader of the Opposition's Address In Reply, Parliament House, Canberra. Canberra: Liberal Party of Australia.

Adler, N. E., \& Ostrove, J. M. (1999). Socioeconomic status and health: What we know and what we don't. Ann N Y Acad Sci, 896, 3-15.

AMWAC. (1997). Characteristics of students entering Australian medical schools 1989 to 1997 (pp. 1-68). Sydney: Australian Institute of Health and Welfare.

Aronson, J., Burgess, D., Phelan, S. M., \& Juarez, L. (2013). Unhealthy interactions: The role of stereotype threat in health disparities. Am J Public Health, 103(1), 50-56. doi: 10.2105/AJPH.2012.300828

Balsa, A. I., \& McGuire, T. G. (2003). Prejudice, clinical uncertainty and stereotyping as sources of health disparities. J Health Econ, 22(1), 89-116.

Beagan, B. L. (2003). Teaching social and cultural awareness to medical students: "it's all very nice to talk about it in theory, but ultimately it makes no difference". Acad Med, 78(6), 605-614.

Beagan, B. L. (2005). Everyday classism in medical school: Experiencing marginality and resistance. Med Educ, 39(8), 777-784. doi: 10.1111/j.13652929.2005.02225.x

Bensing, J. (1991). Doctor-patient communication and the quality of care. Soc Sci Med, 32(11), 1301-1310.

The Bogan Hunters. (2014). Retrieved from http://www.facebook.com/theboganhunters

Burgess, D. J., Fu, S. S., \& van Ryn, M. (2004). Why do providers contribute to disparities and what can be done about it? J Gen Intern Med, 19(11), 11541159. doi: 10.1111/j.1525-1497.2004.30227.x

Campbell, M. (2004). Bogan: Exploring images of Australian cultural marginalisation. University of Melbourne, Parkville.

DeVoe, J. E., Wallace, L. S., \& Fryer, G. E., Jr. (2009). Measuring patients' perceptions of communication with healthcare providers: Do differences in demographic and socioeconomic characteristics matter? Health Expect, 12(1), 70-80. doi: 10.1111/j.1369-7625.2008.00516.x

Dhalla, I. A., Kwong, J. C., Streiner, D. L., Baddour, R. E., Waddell, A. E., \& Johnson, I. L. (2002). Characteristics of first-year students in Canadian medical schools. CMAJ, 166(8), 1029-1035. 
DiMaggio, P. (2012). Sociological perspectives on the face-to-face enactment of class distinction. In S. T. Fiske \& H. R. Markus (Eds.), Facing social class: How societal rank influences interaction (pp. 15-38). New York: Russell Sage Foundation.

Enker, D. (2014, 19 May). Do bogan hunters and what really happens in Bali push an ethical boundary? Sydney Morning Herald. Retrieved from www.smh.com.au/entertainment/tv-and-radio/do-bogan-hunters-and-whatreally-happens-in-bali-push-an-ethical-boundary-20140519-zrh2m.html

Epstein, A. M., Taylor, W. C., \& Seage, G. R., 3rd. (1985). Effects of patients' socioeconomic status and physicians' training and practice on patient-doctor communication. Am J Med, 78(1), 101-106.

Fiske, S. T. (2011). Envy up, scorn down: How status divides us. New York: Russell Sage Foundation.

Fiske, S. T., Cuddy, A. J., \& Glick, P. (2007). Universal dimensions of social cognition: Warmth and competence. Trends Cogn Sci, 11(2), 77-83. doi: 10.1016/j.tics.2006.11.005

Fitzjohn, J., Wilkinson, T., Gill, D., \& Mulder, R. (2003). The demographic characteristics of New Zealand medical students: The New Zealand wellbeing, intentions, debt and experiences (WIDE) survey of medical students 2001 study. N Z Med J, 116(1183), U626.

Geiger, K. A., \& Jordan, C. (2013). The role of societal privilege in the definitions and practices of inclusion. Equality, Diversity \& Inclusion, 33(3), 261-274. doi: 10.1108/EDI-12-2013-0115

Gibson, C. (2013). Welcome to Bogan-ville: Reframing class and place through humour. Journal of Australian Studies, 37(1), 62-75. doi: 10.1080/14443058.2012.756056

Goodman, D. J. (2011). Promoting diversity and social justice: Educating people from privileged groups ( $2^{\text {nd }}$ ed.). New York: Routledge.

Goodman, D. J. (2013). Cultural competency for social justice: A framework for student, staff, faculty, and organizational development (pp. 1-6).

Harris, L. T., \& Fiske, S. T. (2006). Dehumanizing the lowest of the low: Neuroimaging responses to extreme out-groups. Psychol Sci, 17(10), 847853. doi: 10.1111/j.1467-9280.2006.01793.x

Jensen, J. D., King, A. J., Guntzviller, L. M., \& Davis, L. A. (2010). Patient-provider communication and low-income adults: Age, race, literacy, and optimism predict communication satisfaction. Patient Educ Couns, 79(1), 30-35. doi: 10.1016/j.pec.2009.09.041

Kaplan, S. H., Greenfield, S., \& Ware, J. E., Jr. (1989). Assessing the effects of physician-patient interactions on the outcomes of chronic disease. Med Care, 27(3 Suppl), S110-127.

Kelly, P. J. (2012a). Should we rethink how we teach cultural competency in physician assistant education? J Physician Assist Educ, 23(3), 42-45.

Kelly, P. J. (2012b). Using popular movies and reflections to increase the cultural sensitivity of students. J Physician Assist Educ, 23(4), 39-42.

Kennedy, M. (2013). Preparing registrars for the other side of the tracks. Paper presented at the General Practice Education and Training Convention, Perth. Retrived from http://www.onqconferences.com.au/resources/files/gpet2013/abstracts/Mark\% 20Kennedy\%20Preparing.pdf 
Lawler, S. (2005). Disgusted subjects: The making of middle-class identities. The Sociological Review, 53(3), 429-446.

Leigh, A. (2013). Battlers \& billionaires: The story of inequality in Australia. Collingwood: Redback.

Loughnan, S., Haslam, N., Sutton, R. M., \& Spencer, B. (2013). Dehumanization and social class: Animality in the stereotypes of "white trash," "chavs," and "bogans". Social Psychology, Online pub, 45(1), 54-61. doi: 10.1027/18649335/a000159

Major, B., Mendes, W. B., \& Dovidio, J. F. (2013). Intergroup relations and health disparities: A social psychological perspective. Health Psychol, 32(5), 514524. doi: 10.1037/a0030358

Marmot, M. G., Allen, J., Bell, R., \& Goldblatt, P. (2012). Building of the global movement for health equity: From Santiago to Rio and beyond. Lancet, 379(9811), 181-188. doi: 10.1016/S0140-6736(11)61506-7

Marmot, M. G., Smith, G. D., Stansfeld, S., Patel, C., North, F., Head, J., . . Feeney, A. (1991). Health inequalities among British civil servants: The Whitehall II study. Lancet, 337(8754), 1387-1393.

McMahon, N. (2014, 14 May). Bogan Hunters is car-crash TV says creator Paul Fenech. Sydney Morning Herald. Retrieved from http://www.smh.com.au/entertainment/tv-and-radio/bogan-hunters-iscarcrash-tv-says-creator-paul-fenech-20140514-zrc0v.html

NHMRC. (2006). Cultural competency in health: A guide for policy, partnerships and participation (pp. 1-85). Canberra: National Health and Medical Research Council.

Nichols, D. (2011). The bogan delusion: Myths, mischief and misconceptions. Mulgrave: Affirm Press.

OECD. (2014). Society at a glance 2014: OECD social indicators. OECD Publishing. doi: 10.1787/soc_glance-2014-en

Phelan, S. M., Dovidio, J. F., Puhl, R. M., Burgess, D. J., Nelson, D. B., Yeazel, M. W., . . . van Ryn, M. (2013). Implicit and explicit weight bias in a national sample of 4,732 medical students: The medical student CHANGES study. Obesity (Silver Spring). doi: 10.1002/oby.20687

Phillips, A. (2010). What's wrong with essentialism? Distinktion, 20, 47-60.

Pini, B., \& Previte, J. (2013). Gender, class and sexuality in contemporary Australia. Australian Feminist Studies, 28(78), 348-363. doi: 10.1080/08164649.2013.857385

Raingruber, B. (2014). Health disparities. In B. Raingruber (Ed.), Contemporary health promotion in nursing practice $\left(1^{\text {st }}\right.$ ed., pp. 123-160). Burlington, MA: Jones \& Bartlett Learning.

Ridgeway, C. L., \& Fisk, S. R. (2012). Class rules, status dynamics, and "gateway" interactions. In S. T. Fiske \& H. R. Markus (Eds.), Facing social class: How societal rank influences interaction (pp. 131-151). New York: Russell Sage Foundation.

Rossiter, P. (2013). Bogans: A sticky subject. Continuum: Journal of Media \& Cultural Studies, 27(1), 80-92. doi: 10.1080/10304312.2013.737198

Rudman, L. A. (2004). Social justice in our minds, homes, and society: The nature, causes, and consequences of implicit bias. Soc Jus Res, 17(2), 129-142.

Skeggs, B. (2011). Imagining personhood differently: Person value and autonomist working-class value practices. The Sociological Review, 59(3), 496-513. 
Spencer, B., \& Castano, E. (2007). Social class is dead. Long live social class! Stereotype threat among low socioeconomic status individuals. Soc Jus Res, 20, 418-432.

Stephens, N. M., Fryberg, S. A., \& Markus, H. R. (2012). It's your choice: How the middle-class model of independence disadvantages working-class Americans. In S. T. Fiske \& H. R. Markus (Eds.), Facing social class: How societal rank influences interaction (pp. 87-106). New York: Russell Sage Foundation.

UA. (2008). Advancing equity and participation in Australian higher education: Action to address participation and equity levels in higher education of people from low socioeconomic backgrounds and Indigenous people. Equity and Participation Action Plan. Canberra: Universities Australia.

van Ryn, M., \& Burke, J. (2000). The effect of patient race and socio-economic status on physicians' perceptions of patients. Soc Sci Med, 50(6), 813-828.

VEOHRC. (2014). Guideline for general practices: Complying with the Equal Opportunity Act 2010 when providing services. In V. E. O. a. H. R. Commission (Ed.). Carlton.

Verlinde, E., De Laender, N., De Maesschalck, S., Deveugele, M., \& Willems, S. (2012). The social gradient in doctor-patient communication. Int $J$ Equity Health, 11:12, 1-14. doi: 10.1186/1475-9276-11-12

Voss-DeMeester, R. H., McCullough, K. W., Cook, S. C., El-Shamaa, M., \& Chin, M. $\mathrm{H}$. (2014). Responding to culture: Beyond cultural competence training. Finding Answers: Disparities Research for Change (pp. 14). Chicago: Robert Wood Johnson Foundation.

Wear, D., \& Kuczewski, M. G. (2008). Perspective: Medical students' perceptions of the poor: What impact can medical education have? Acad Med, 83(7), 639645. doi: 10.1097/ACM.0b013e3181782d67

Willems, S., De Maesschalck, S., Deveugele, M., Derese, A., \& De Maeseneer, J. (2005). Socio-economic status of the patient and doctor-patient communication: Does it make a difference? Patient Educ Couns, 56(2), 139146. doi: 10.1016/j.pec.2004.02.011

Woo, J. K., Ghorayeb, S. H., Lee, C. K., Sangha, H., \& Richter, S. (2004). Effect of patient socioeconomic status on perceptions of first- and second-year medical students. CMAJ, 170(13), 1915-1919.

\section{Biographical notes}

Dr Mark Kennedy has been a general practitioner in the under-resourced northern suburbs of Geelong, Victoria, for 25 years. He is currently Medical Director of Northern Bay Health, a network of three general practices and a diabetes centre. He is an Honorary Clinical Associate Professor and is involved in primary care diabetes research at the Melbourne University Department of General Practice. Mark has been involved in teaching medical students and registrars for almost 20 years and has special interests in managing chronic disease and in the impact of health literacy and socio-economic disadvantage on health outcomes. 
Maeve Kennedy is a Research Assistant at Northern Bay Health and she holds a Master of Development Studies (Gender \& Development). She is interested in the interlinkages between different dimensions of social exclusion, especially class and gender, and the effects these have on people's health and wellbeing. She is currently undertaking postgraduate study in sustainable agriculture, to further investigate the links between food systems, health, and sustainable development. 